\title{
Impacto de la Salud Oral en la Calidad de Vida de Adultos Mayores
}

\author{
Shyrley Díaz Cárdenas ${ }^{a}$, Katherine Arrieta Vergara ${ }^{\text {, Ketty Ramos Martínez }}{ }^{\mathrm{a}}$
}

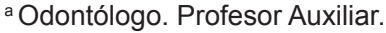
Departamento de Odontología Preventiva y Social. Universidad de Cartagena (Colombia).

b Odontólogo. Profesor Asistente y Jefe del Departamento de Odontología Preventiva y Social. Universidad de Cartagena (Colombia).
\end{abstract}

Correspondencia: Shyrley Díaz Cárdenas,

Universidad de Cartagena,

Campus de la Salud

Zaragocilla, Facultad de

Odontología-Departamento de Investigaciones.

Telf.: 6698184 ext. 110.

E-mail: shyrley77@hotmail.com.

Recibido el 27 de diciembre de 2011.

Aceptado para su publicación el 10 de enero de 2012.

\section{RESUMEN}

Objetivo. Asociar salud bucal y calidad de vida en adultos mayores atendidos en la Facultad de Odontología, Universidad de Cartagena.

Diseño. Estudio de corte transversal.

Emplazamiento. Municipio de Cartagena de Indias, Colombia.

Participantes. Censo de 120 adultos mayores, primer periodo de 2011.

Mediciones Principales. Se evaluaron variables socio demográficas, familiares, índice de Salud Oral Geriátrico (GOHAl) y diagnostico de salud bucal. Los datos fueron analizados a partir de proporciones, prueba $\chi^{2}$ para la comprobación de asociaciones estadísticamente significativas y razones de disparidad (OR).

Resultados. Al realizar la prueba $\chi^{2}$ se encontró significación estadística entre una salud bucal deficiente y el impacto negativo sobre la calidad de vida, según GOHAI $(p=0,01)$ y al asociar este ultimo y los problemas de origen endodóntico (OR: 3,5; IC 95\%: 1,4-8,4; $p=0,004$ ), hipertensión arterial (OR: 4,44; IC 95\%:1,5-12; $p=0,005$ ) y vivir con más de 5 miembros en la familia (OR: 3,38 ; IC 95\%: 1,34-8,49; $p=0,009$ ). En la regresión logística estos factores constituyeron el modelo que mejor explicaba el detrimento en la calidad de vida relacionada con la salud bucal $\left(p=0,0001 ; x^{2}=21,31\right)$

Conclusión. Los problemas endodónticos comprometen la calidad de vida en los adultos mayores. Es necesario un enfoque psicosocial de la salud bucal que incluya otros factores capaces de comprometer el bienestar.

Palabras clave. Ancianos, Calidad de Vida, Salud Bucal.

\section{ABSTRACT}

Impact of Oral Health on Quality of Life in Older Adults

Objective. To relate oral health to quality of life in older adult patients at the Faculty of Dentistry, University of Cartagena.

Design. Cross sectional study.

Location. Municipality of Cartagena de Indias, Colombia.

Participants. 120 older adults selected from census of first quarter of 2011.

Main Measures. Sociodemographic variables, family life, Geriatric Oral Health Index (GOHAI) and diagnosis of oral health. Data were analyzed using proportions, chi-square test for association between variables and odds ratios (OR).

Results. Chi-square test revealed statistical significance between poor oral health and negative impact on quality of life, according to the GOHAI ( $p=0.01)$. Problems of endodontic origin (OR: $3.5,95 \% \mathrm{Cl}: 1.4$ to $8.4, \mathrm{p}=0.004$ ), hypertension (OR: $4.44, \mathrm{Cl} 95 \%: 1.5$ to $12, \mathrm{p}=0.005$ ) and living in households with more than 5 family members (OR: $3.38,95 \%: 1.34$ to $8.49, p=0.009$ ) all had negative impact on quality of life. In the logistic regression, these factors best explained the decreased quality of life associated with oral health $(p=0.0001, x 2=21.31)$.

Conclusion. Endodontic problems compromise quality of life in older adults. A psychosocial approach to oral health is needed that includes other factors capable of jeopardizing well-being. Key Words: Aged, Quality of Life, Oral Health.

\section{INTRODUCCIÓN}

En el adulto mayor se ve reflejado el comportamiento saludable que se adquirió en las primeras etapas del ciclo vital individual. En el envejecimiento se generan una serie de cambios en el estado social, en la percepción sensorial y en las funciones cognitivas y motoras de los individuos ${ }^{1,2}$. A nivel de salud bucal también se presentan cambios sobre los tejidos orales y funciones, y cambios secundarios a factores extrínsecos, incrementándose la pérdida de dientes debido a la enfermedad periodontal, caries y lesiones de la mucosa oral ${ }^{2}$. La falta de dientes y ausencia de prótesis dental tienen 
una relación directa con la salud, debido a que al realizar inadecuadamente la función masticatoria, sobre todo de alimentos de consistencia dura, se producen trastornos de la nutrición. Además se afecta la autorrealización y aceptación al generarse vergüenza, baja autoestima, dolor e incomodidad frente a otras personas durante el momento de las comidas y en los momentos de socialización ${ }^{3}$. Otros factores generales como el uso de medicamentos, altos costes de la atención en salud, el nivel educativo y la clase social pueden comprometer la salud del adulto mayor ${ }^{2}$. La calidad de vida relacionada con la salud oral ha sido estudiada principalmente en los adultos mayores, probablemente debido al interés general por este grupo de edad, el cual ha presentado un acelerado crecimiento en las últimas décadas en la mayoría de los países ${ }^{4}$.

Se ha utilizado el Índice de Salud Oral Geriátrico (GOHAI) como una herramienta para la identificación del impacto de la salud oral en la calidad de vida de los adultos mayores, evidenciando que una baja auto percepción de calidad de vida se encuentra directamente relacionada con un pobre estado de salud bucal ${ }^{5}$. Descrita por Atchison y Dolan ${ }^{5}$, y como fundamento para el desarrollo del GOHAI, la salud oral fue definida como la ausencia de dolor e infección compatible con una dentición funcional y cómoda que permita al individuo continuar en su rol social ${ }^{6}$. Algunos estudios revelan que la buena higiene oral puede mejorar la calidad de vida de las personas ${ }^{7,8}$.

El objetivo del estudio fue describir la percepción de calidad de vida relacionada con la salud bucal en los sujetos adultos mayores atendidos en las clínicas odontológicas de la Universidad de Cartagena, así como conocer los factores específicos a nivel de salud oral que ejercen un impacto en dicha calidad de vida y pueden ser modificados y controlados para mejorar los programas de promoción y prevención bucal en esta población.

\section{MATERIAL Y MÉTODOS}

Estudio de corte transversal en la ciudad de Cartagena de Indias, cuyos sujetos de estudio fueron 120 pacientes adultos mayores de 60 años que asistieron a las Clínicas Integrales del Adulto en la Facultad de Odontología de la Universidad de Cartagena, seleccionados de enero a abril de 2011. Se tuvieron en cuenta todos los adultos mayores que aceptaran participar en el estudio y que no presentaron una discapacidad mental y sensorial motora.
Para el inicio de las mediciones se solicitó el consentimiento informado por escrito de los pacientes, teniendo en cuenta las disposiciones internacionales: Declaración de Helsinki (modificación de Edimburgo 2000) y la normativa para Colombia (resolución 8.430 de 1993 del antiguo Ministerio de la Salud).

La recolección de datos fue realizada en dos etapas: en primer lugar, se realizaron los exámenes clínicos en las unidades odontológicas para obtener el diagnóstico del estado de salud bucal, aplicándose el Índice de Salud Oral Geriátrico (GOHAI), que evaluaba los niveles de percepción de Calidad de Vida relacionados con la salud bucal en los adultos mayores, presencia de caries y cálculos dentales, restos radiculares, obturaciones, presencia y estado de prótesis total y removible y lesiones en la mucosa y su etiología; en una segunda etapa se aplicó la encuesta estructurada (habiéndose evaluado la validez de apariencia a partir de dos jueces en un prueba piloto previa para valorar su comprensión y confrontar las diferentes puntuaciones obtenidas con la teoría), a través de visitas domiciliarias para obtener las variables sociodemográficas y familiares, conteniendo 14 preguntas con una sola opción de respuesta e incluyendo información sobre edad, sexo, estrato socioeconómico, nivel de escolaridad, estado civil, tipo de religión y ocupación, variables familiares, como composición del hogar, tipo y tenencia de la vivienda, y funcionalidad familiar evaluada a través del cuestionario Apgar Familiar.

Para el análisis estadístico se calcularon promedios, desviación estándar, distribución de frecuencias y porcentajes. Posteriormente se estimó la ocurrencia del estado de salud bucal y los niveles de percepción de calidad de vida relacionada con la salud bucal mediante prevalencias, calculando intervalos de confianza del $95 \%$. Se evaluaron las relaciones entre variables utilizando la prueba Chi cuadrado para comprobar la significación estadística, con un límite de decisión de 0.05 . Todos los valores de los estimadores fueron ajustados a partir del diseño muestral. La fuerza de asociación se estimó mediante OR con intervalos de confianza al 95\%. Además, para el análisis multivariable se utilizó la regresión logística nominal, teniendo en cuenta las variables que marcaron una probabilidad igual o menor al $5 \%$. Para el análisis se utilizó el programa estadístico STATA versión para Windows 10.0.

\section{RESULTADOS}

El promedio de edad de los participantes fue de 68,3 años (DE: 6,3). La tabla 1 muestra las 


\begin{tabular}{l|cc}
\hline VARIABLES SOCIODEMOGRÁFICAS & $\begin{array}{c}\text { FRECUENCIA } \\
\mathbf{n = 1 2 0}\end{array}$ & $\begin{array}{c}\text { PORCENTAJE } \\
\%\end{array}$ \\
\hline SEXO & & \\
- Femenino & 68 & 56,6 \\
- Masculino & 52 & 43,3 \\
OCUPACIÓN & & \\
- Empleado & 26 & 21,6 \\
- Desempleado & 34 & 28,3 \\
- Pensionado & 39 & 32,5 \\
- Otros & 21 & 17,5 \\
SEGURIDAD SOCIAL & 62 & 51,6 \\
- Contributivo & 55 & 45,8 \\
- Subsidiado & 3 & 2,5 \\
- Régimen Especial & & \\
ESTRATO SOCIOECONÓMICO & 37 & 30,8 \\
- 1 & 39 & 32,5 \\
- 2 & 43 & 35,8 \\
- 3 & 1 & 0,8 \\
- 4 & & \\
INGRESOS SOCIOECONÓMICOS & 53 & 44,16 \\
- Hasta 1 SMMLV & 43 & 35,8 \\
- Más de 1 a 2 SMMLV & 22 & 18,3 \\
- Más de 2 a 3 SMMLV & 2 & 1,6 \\
- Más de 3 a 4 SMMLV & & \\
\hline
\end{tabular}

Tabla 1. Variables sociodemográficas de los sujetos de estudio.

\begin{tabular}{l|ccc}
\hline \multicolumn{1}{c|}{ DIAGNOSTICO DE SALUD BUCAL } & $\begin{array}{c}\text { FRECUENCIA } \\
\mathbf{n = 1 2 0}\end{array}$ & $\begin{array}{c}\text { PORCENTAJE } \\
\%\end{array}$ & IC 95\% \\
\hline Motivo de consulta & & & \\
- Origen infeccioso & 29 & 24,1 & $16,3-31,9$ \\
- Origen traumático (uso de prótesis desadaptadas) & 102 & 85 & $78,5-91,4$ \\
- Origen endodóntico & 54 & 45 & $35,9-54,0$ \\
- Origen periodontal & 93 & 77,5 & $69,9-85,0$ \\
Presencia de Menos de 19 dientes & 90 & 75 & $67,1-82,8$ \\
Presencia de prótesis dental & 72 & 60 & $51,1-68,8$ \\
Prótesis fija & 20 & 16,6 & $9,9-23,4$ \\
Prótesis removible & 47 & 39,1 & $30,3-48,0$ \\
Prótesis total & 36 & 30 & $21,6-38,3$ \\
Prótesis desadaptada & 71 & 59,7 & $50,2-68,0$ \\
Cálculos dentales & 96 & 80 & $72,7-87,2$ \\
Restos radiculares & 28 & 23,3 & $15,6-31,0$ \\
Dientes con caries & 87 & 72,5 & $64,3-80,6$ \\
Obturaciones & 71 & 59,7 & $50,2-68,0$ \\
Obturaciones desadaptadas & 58 & 48,3 & $39,2-57,4$ \\
Problemas de articulación temporomandibular & 90 & 75 & $27,9-45,4$ \\
& & & \\
\hline
\end{tabular}

Tabla 2. Diagnostico de salud bucal de los sujetos de estudio. 
Índice de Salud Oral Geriátrico (GOHAI)

\begin{tabular}{|c|c|c|c|c|c|c|}
\hline & $\begin{array}{l}\text { NUNCA } \\
\mathbf{N}^{\circ}(\%)\end{array}$ & $\begin{array}{c}\text { RARA } \\
\text { VEZ } \\
N^{\circ}(\%)\end{array}$ & $\begin{array}{c}\text { A } \\
\text { VECES } \\
N^{\circ}(\%)\end{array}$ & $\begin{array}{c}\text { A } \\
\text { MENUDO } \\
\mathrm{N}^{\circ}(\%)\end{array}$ & $\begin{array}{c}\text { MUY A } \\
\text { MENUDO } \\
\mathbf{N}^{\circ}(\%)\end{array}$ & $\begin{array}{l}\text { SIEMPRE } \\
\mathrm{N}^{\circ}(\%)\end{array}$ \\
\hline \multicolumn{7}{|l|}{ FUNCIÓN FÍsıCA } \\
\hline $\begin{array}{l}\text { ¿Limitó la clase o cantidad de alimentos debido a problemas con sus } \\
\text { dientes o prótesis dentales? }\end{array}$ & $3(2,5)$ & $4(3,3)$ & $9(7,5)$ & $34(28,3)$ & $49(40,8)$ & $21(17,5)$ \\
\hline ¿Pudo tragar cómodamente? & $5(4,1)$ & $76(63,3)$ & $29(24,1)$ & $6(5,0)$ & $1(0,8)$ & $3(2,5)$ \\
\hline $\begin{array}{l}\text { ¿Sus dientes o prótesis dentales le impidieron hablar del modo que } \\
\text { usted quería? }\end{array}$ & $3(2,5)$ & $4(3,3)$ & $22(18,3)$ & $42(35,0)$ & $36(30,0)$ & $13(10,8)$ \\
\hline $\begin{array}{l}\text { ¿Pudo comer lo que usted quería sin sentir molestias de sus dientes } \\
\text { o prótesis dentales? }\end{array}$ & $8(6,6)$ & $75(6,25)$ & $27(22,5)$ & $3(2,5)$ & $6(5,0)$ & $1(0,8)$ \\
\hline \multicolumn{7}{|l|}{ FUNCIÓN PSICOSOCIAL } \\
\hline $\begin{array}{l}\text { ¿Evitó estar en contacto con personas debido a la condición de sus } \\
\text { dientes o prótesis dentales? }\end{array}$ & $6(5,0)$ & $9(7,5)$ & $14(11,6)$ & $43(35,8)$ & $43(35,8)$ & $5(4,1)$ \\
\hline $\begin{array}{l}\text { ¿Se sintió satisfecho o contento con la apariencia de sus dientes, } \\
\text { encías o prótesis dentales? }\end{array}$ & $12(10,0)$ & $46(38,3)$ & $11(9,1)$ & $29(24,1)$ & $14(11,6)$ & $8(6,6)$ \\
\hline $\begin{array}{l}\text { ¿Se preocupó o intranquilizó por problemas con sus dientes, encías } \\
\text { o prótesis dentales? }\end{array}$ & $0(0,0)$ & $3(2,5)$ & $8(6,6)$ & $31(25,8)$ & $51(42,5)$ & $27(22,5)$ \\
\hline $\begin{array}{l}\text { ¿Se sintió nervioso o consiente debido a problemas con sus dientes, } \\
\text { encías prótesis dentales? }\end{array}$ & $2(1,6)$ & $2(1,6)$ & $10(8,3)$ & $29(24,1)$ & $48(40,0)$ & $29(24,1)$ \\
\hline $\begin{array}{l}\text { ¿Se sintió incómodo al comer frente a otras personas debido a } \\
\text { problemas con sus dientes, encías o prótesis dentales? }\end{array}$ & $5(4,1)$ & $3(2,5)$ & $11(9,1)$ & $32(26,6)$ & $53(44,1)$ & $16(13,3)$ \\
\hline $\begin{array}{l}\text { ¿Evitó reírse o sonreírse debido a que sus dientes o prótesis } \\
\text { dentales eran antiestéticos? }\end{array}$ & $3(2,5)$ & $5(4,1)$ & $10(8,3)$ & $35(29,1)$ & $46(38,3)$ & $21(17,5)$ \\
\hline \multicolumn{7}{|l|}{ FUNCIÓN DOLOR } \\
\hline ¿Ha tenido dolor o molestias alrededor de la boca? & $0(0,0)$ & $1(0,8)$ & $4(3,3)$ & $50(41,6)$ & $42(35,0)$ & $23(19,1)$ \\
\hline $\begin{array}{l}\text { ¿Sintió sus dientes o encías sensibles a los alimentos calientes, fríos } \\
\text { o dulces? }\end{array}$ & $0(0,0)$ & $1(0,8)$ & $9(7,5)$ & $51(42,5)$ & $33(27,5)$ & $26(21,6)$ \\
\hline
\end{tabular}

Tabla 3. Función física, psicosocial y sensibilidad al dolor producidos por problemas bucales según el GOHAI.

características sociodemográficas de los sujetos de estudio.

En cuanto a las variables familiares, se observó que el principal cuidador de los adultos mayores fue el cónyuge con un 49,1\% (IC 95\%: 40,0 - 58,2), el tipo de familia predominante fue la nuclear $(45,8 \%$; IC $95 \%: 36,7-54,8)$ y la mayoría de las familias estaban integradas por 2 y 3 miembros (25\%; IC 95\%: 17,1-32,8), viviendo la mayoría en viviendas tipo casa $(77,5 \%$; IC $95 \%$ : $69,9-85,0)$, las cuales eran propias en el $71,6 \%$ de los casos (IC 95\%: 63,4-79,8). A través del Apgar Familiar, se encontró disfuncionalidad familiar en un $55 \%$ (IC 95\%: 45-64). Con relación a los antecedentes médicos familiares y personales, la hipertensión estaba presente en el 70,8\% (IC 95\%: 62,5 - 79,0) y en el $61,6 \%$ (IC 95\%: 52,8- 70,5) respectivamente, seguida de la diabetes (33,3\%; IC 95\%: $24,7-41,8)$ $\mathrm{y}$, con menor frecuencia, los problemas respiratorios (10\%; IC 95\%:15,4 - 45,5).

Con respecto al diagnóstico de salud bucal, el $75 \%$ de los participantes presentaban menos de 19 dientes, el $59,1 \%$ y el $48,3 \%$ presentaban prótesis y restauraciones desadaptadas, respectivamente, el $80,0 \%$ presentaba cálculo dental, el $23,3 \%$ restos radiculares, el $72,5 \%$ caries dental y el $75 \%$ problemas de articulación temporo-mandibular. Los motivos de consulta más presentes fueron los de origen traumático y periodontal, en $85 \%$ y $77,5 \%$ respectivamente (tabla 2 ). 
PERCEPCION DE LA CALIDAD DE VIDA SEGÚN EL GOHAI

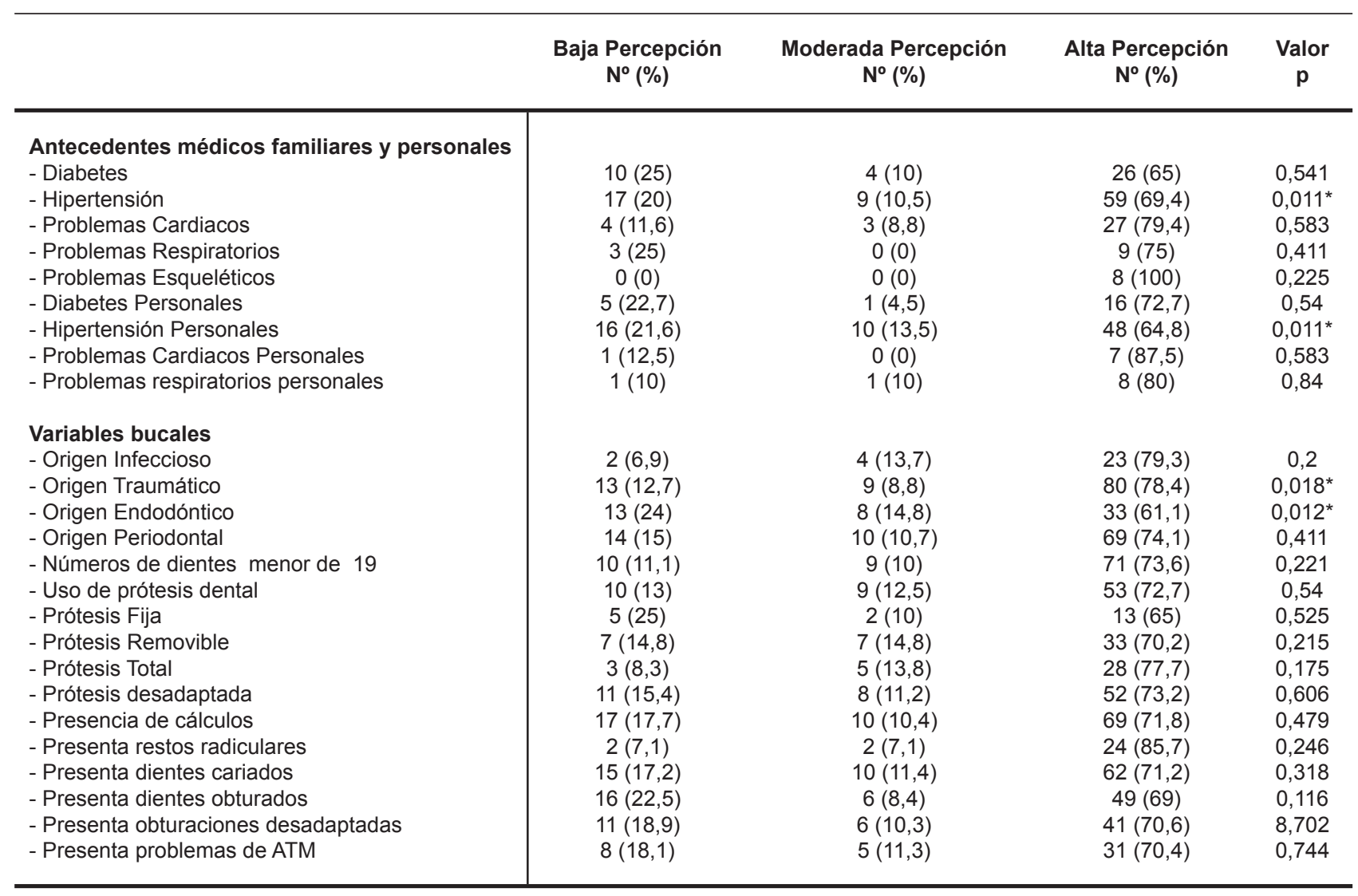

Tabla 4. Relación de los antecedentes médicos (familiares y personales) y del estado bucal con la percepción de la calidad de vida. *valores de p inferiores a 0,05. ATM: articulación temporo-mandibular.

\begin{tabular}{l|ccc}
\hline \multicolumn{1}{c|}{ Características } & $\begin{array}{c}\text { Univariado } \\
\text { OR (IC 95\%) }\end{array}$ & $\begin{array}{c}\text { Valor } \\
\mathbf{p ~}\end{array}$ & $\begin{array}{c}\text { Multivariado } \\
\text { OR* (IC 95\%) }\end{array}$ \\
\hline Más de 5 miembros en la familia & $3,38(1,34-8,49)^{* *}$ & 0,009 & $2,94(1,09-7,95)$ \\
Funcionalidad familiar & $0,34(0,14-0,79) \dagger$ & 0,01 & - \\
Hipertensión & $4,44(1,5-12)^{*}$ & 0,005 & $3,83(1,29-11,36)$ \\
Origen Endodóntico & $3,5(1,4-8,4)$ a & 0,004 & $3,21(1,28-8,02)$ \\
Origen Traumático & $0,27(0,097-0,77) \dagger$ & 0,01 & - \\
Menos de 19 dientes & $0,4(0,16-0,97) \ddagger$ & 0,04 & - \\
\hline
\end{tabular}

Tabla 5. Asociación entre el Índice Geriátrico de Salud Oral y las variables socio demográficas y el estado de salud bucal de los sujetos de estudio. ${ }^{*}$ Estimadores ajustados por regresión logística (Chi-cuadrado $=21,31 ; p=0.0001$ ). 
Al analizar los dimensiones evaluadas por el Índice de Salud Oral Geriátrico (GOHAI), que relacionan la salud bucal con la calidad de vida, se encontró que la función psicosocial fue el componente reportado con mayor frecuencia, en especial aquél relacionado con la incomodidad que presentan los adultos mayores al comer frente a otras personas por problemas con sus dientes (44\%). Con respecto a la función física, el 63,3\% manifestó incomodidad al deglutir sus alimentos $\mathrm{y}$, con respecto a la sensibilidad dental, la molestia más percibida fue la sensibilidad a los alimentos calientes, fríos o dulces, en un $42,5 \%$. (tabla 3 ). Con respecto a los niveles de percepción de calidad de vida relacionada con la salud bucal, a través del índice del GOHAl, el 74,1\% (IC 95\%: 66,2 - 82,1) de los participantes manifestaron un alto impacto de su salud bucal sobre su calidad de vida. Sólo el 16,6\% (IC 95\%: $9,9-23,4)$ tuvo una percepción baja y el 9,7\% (IC 95\%: $3,9-14,4)$ una percepción moderada.

Al relacionar la percepción de la calidad de vida con las variables sociodemográficas y familiares, sólo se encontró significación estadística con los antecedentes familiares y personales de hipertensión arterial $(p=0,01)$ (tabla 4$)$. Con respecto a los diagnósticos de salud bucal, al ser relacionadas con los niveles de percepción de calidad de vida, se encontró significación estadística para los diagnósticos de origen traumático y de origen endodóntico $(p=0,01)$ y al relacionar la calidad de vida con la presencia de menos de 19 dientes $(p=$ 0,01 ) (tabla 4). Al estimar los OR, se encontró una asociación entre el impacto de la salud bucal sobre la calidad de vida y el hecho de vivir con más de 5 miembros en las familias, presentar hipertensión arterial y los diagnósticos de origen endodóntico $(p<0,05)$, comportándose éstos como factores de riesgo para la calidad de vida de las personas (tabla 5). En el análisis de regresión logística, el modelo que mejor explica el detrimento en la calidad de vida relacionada con la salud bucal en los sujetos de estudio fue el conformado por los siguientes factores: convivir con más de 5 miembros en las familias, el antecedente de hipertensión arterial y los diagnósticos de origen endodóntico. El modelo fue estadísticamente significativo $\left(p=0,0001 ; x^{2}=\right.$ 21,31) (tabla 5).

\section{DISCUSIÓN}

Existen muchos estudios que relacionan el impacto de la salud bucal sobre la calidad de vida de los adultos mayores. En México, Esquivel y Jiménez ${ }^{9}$ encontraron que el nivel de escolaridad más frecuente fue la primaria completa, exclusivamente en mujeres, similar a los resultados obtenidos en este estudio y a los hallazgos confirmados por Monge, quien reporta a nivel mundial que las mujeres presentan una mayor expectativa de vida que el hombre, a pesar de ser el género que más problemas padece surgidos por su historia personal, como mala nutrición, embarazos y violencia intrafamiliar, entre otros ${ }^{10}$. A esto se suma que la baja escolaridad encontrada es una respuesta social al poco acceso presentado hace 60 años a servicios como la salud, el trabajo y la educación, y sobre todo a la poca participación de la mujer a partir de nuevos roles en la sociedad.

Según los resultados del presente estudio, el 32\% de los participantes correspondían a pensionados, con ingresos menores de un salario mínimo legal vigente (SMMLV) y de estrato socioeconómico bajo, lo que podría limitar el mantenimiento y la satisfacción de sus necesidades básicas, como alimentación, salud y vivienda. Un punto a favor en este estudio es el hecho de que esta población vivía en casas propias, lo que genera no sólo menos egresos económicos sino una estabilidad emocional en esta etapa. Coronado et al, encontraron en adultos mayores de Chiclayo (Perú) que la mayor parte de éstos no ejercían una actividad laboral, debido a que eran pensionados, siendo además uno de los grupos sociales con más bajos ingresos socioeconómicos ${ }^{11}$. En este sentido, la diferencia entre vejez saludable y un adulto mayor enfermo está en la cantidad de dinero disponible según algunos autores, por lo que desde la etapa de adulto joven se debe visionar la adquisición de recursos económicos que puedan proveer cuidados dignos en edades mayores que conlleven a una buena calidad de vida ${ }^{12}$.

Por otra parte, un factor encontrado en el estudio, y reportado en la literatura como factor protector, fue que la gran mayoría de adultos mayores pertenecían a la religión católica, aunque no basta con pertenecer sino también conocer su actividad en cuanto a las prácticas religiosas. Por lo general este grupo busca también apoyo no sólo en la familia sino en los amigos como soporte social extra familiar y la religión es una buena estrategia para buscar este apoyo. Además, en esta etapa se genera un reencuentro con la vida más espiritual, probablemente por la cercanía a la muerte, lo que conlleva mayor unión a la religión ${ }^{13}$. Con respecto a la funcionalidad familiar, Díaz y Soler ${ }^{14}$ encontraron una buena percepción de la funcionalidad en las 
familias de los adultos mayores al ser evaluadas por el Apgar Familiar en la comunidad rural del Policlínico Docente de San Cristóbal, Cuba, hallazgos similares a los observados en el presente estudio, que confirman que, si bien la familia es muy importante en cualquier etapa de la vida, para el adulto mayor es cada día más importante, debido a su creciente dependencia y a la pérdida progresiva de contactos sociales.

En cuanto a los antecedentes médicos familiares y personales, en el presente estudio se encontró que las patologías de mayor prevalencia fueron la hipertensión arterial, la diabetes y, en menor proporción, los problemas respiratorios, datos igualmente reportados por Peña et al en México, donde además reportaron la insuficiencia renal como antecedente sistémico médico padecido por el adulto mayor ${ }^{15}$. Esto confirma las causas actuales de morbilidad y mortalidad de nuestro país, Colombia ${ }^{16}$. Estas patologías crónicas, que originan discapacidad y minusvalía, deben ser correlacionadas con la calidad de vida y los años de vida saludable. Según Milton Terris, aunque hace unas décadas las personas vivían menos años, su vida era de buena calidad; en la actualidad, el número mayor de años vividos y las diversas formas de prolongación de la vida, la mantienen, pero no garantizan la calidad de vida ${ }^{13}$.

Con respecto al estado de salud bucal de los adultos mayores, los motivos de consulta más reportados fueron aquéllos de origen traumático, endodóntico y periodontal, etiologías que generan dolor. La gran mayoría de los participantes eran desdentados parciales, con menos de 19 dientes presentes en boca, presencia de cálculos dentales y de caries; usaban prótesis parcial removible, pero en su gran mayoría desadaptadas. Estas condiciones fueron muy similares a lo reportado por Pinzón et al en España ${ }^{17}$, donde los adultos mayores presentaban enfermedad periodontal y mala higiene bucal, caries dental, necesitaban prótesis dentales y presentaban problemas en la función masticatoria.

Con respecto al impacto de la salud bucal sobre la percepción de la calidad de vida, se reportaron mayores puntuaciones en la dimensión que evaluaba la función psicosocial, al no sentirse cómodos al comer delante de otras personas por problemas con sus dientes, comparada con la función física y la sensibilidad al dolor. Así mismo, estas dimensiones o dominios se han evaluado en otros estudios, los cuales mostraron más comprometidos los aspectos funcionales y psicosociales sobre la salud bucal ${ }^{8,9}$.
Los resultados obtenidos describen que los adultos mayores perciben que sus problemas dentales de origen endodóntico o traumático, que son acompañados de dolor, y la ausencia de la gran mayoría de sus dientes representan un impacto negativo en su calidad de vida, confirmando la importancia de la salud bucal como factor determinante para la consecución del bienestar general. En este sentido, aunque los problemas dentarios o los desórdenes bucales de cualquier naturaleza ofrezcan poco riesgo para la vida, éstos influyen determinantemente en la calidad de vida de los individuos. El hecho de no haberse prestado atención a los posibles efectos en la salud general de los desórdenes bucales se debe básicamente a la separación histórica entre la Medicina y la Odontología ${ }^{18,19,20}$. Al realizar la asociación a través de los estimadores OR, se encontró una asociación entre los problemas a nivel bucal y el impacto que ejercen estos problemas en la calidad de vida de los adultos mayores, con un OR de 3,5, al igual que Mesas et al, quienes encontraron un OR de 3,41 al asociar los problemas de salud bucal con una percepción negativa sobre la calidad de vida de adultos mayores de un programa de salud familiar en Londrina, Brasil, en $2010^{3}$.

Se hace indispensable, por tanto, el trabajo con la población joven en la promoción de estilos de vida saludables que les permita disfrutar de su etapa de adulto mayor con una buena calidad de vida. Además, se está promoviendo el intercambio de información entre el odontólogo y otros profesionales de la salud como la base de un sistema eficiente de salud pública y del cuidado de la salud, lo que implica que el estomatólogo debe involucrarse activamente con sus pares en otros ámbitos del cuidado de la salud general, fomentando el abordaje multidisciplinario de las necesidades de atención de los adultos mayores ${ }^{21}$.

En esta investigación se confirma la influencia de la salud bucal en la calidad de vida de los adultos mayores. Las dimensiones registradas con mayor impacto son malestar psicológico, dolor físico y limitación funcional, lo cual indica implicaciones importantes en la realización de sus actividades cotidianas, por lo que las personas con estas características acuden a estos centros a solicitar el servicio odontológico, por presentar problemas en su capacidad fisiológica bucodental, que además les impide mantener una comunicación social adecuada. Se recomienda entonces que estas necesidades sean evaluadas antes de que afecten su calidad de vida, sin embargo, debido a la creciente 
demanda de atención de este grupo poblacional en nuestro país, se genera un importante problema de salud pública que debe ser abordado.

\section{BIBLIOGRAFÍA}

1. Barrantes M. Género, vejez y salud. Acta Bioethica. 2006; 12:193-7.

2. Colussi CF, De Freitas SF, Calvo MC. The prosthetic need WHO index: a comparison between self-perception and professional assessment in an elderly population. Gerodontology. 2009; 26:187-92.

3. Mesas AM, Andrade SM, Cabrera MA, Bueno VL. Oral health status and nutritional deficit in noninstitutionalized older adults in Londrina, Brazil Rev Bras Epidemiol. 2010; 13:434-45.

4. World Population Ageing 1950-2050. United Nations; 1995. Disponible en: http://www.un.org/esa/population/ publications/worldageing19502050/pdf/preface web.pdf.

5. Atchison KA, Dolan TA. Development of the Geriatric Oral Health Assessment Index. J Dent Educ. 1990; 54:680-7.

6. Tubert-Jeannin S, Riordan PJ, Morel-Papermont A, Porchera S, Saby-Collet S. Community Dent Oral Epidemiol. 2003; 31: 275-84.

7. Restrepo SL, Morales RM, Ramírez MC, López MV, Varela LE. Los hábitos alimentarios en el adulto mayor y su relación con los procesos protectores y deteriorantes en salud. Rev Chil Nutr. 2006; 33:500-10.

8. Bortoletto A, Oliveira T. A cross-sectional study of oral health-related quality of life of Piracicaba's elderly population. Rev Odonto Ciênc. 2010; 25:126-31.

9. Esquivel R, Jimenez J. Necesidades de Atención Odontológica en Adultos Mayores Mediante la Aplicación del GOHAI. Revista ADM. 2010; 57; 127-31.

10. Monge M. Género, Vejez y Salud. Acta Bioethica. 2006; 6:193-7.
11. Coronado J, Díaz C. Percepción de la Calidad de Vida Relacionada con Salud de Adultos Mayores Residentes en la Ciudad de Chiclayo. Acta Medica Per. 2009: 26:230-8.

12. Wong $R$, Espinoza M. Adulto Mayor Mexicano en el Contexto Socioeconómico Amplio: Salud y Envejecimiento. Salud Pública de México. 2007; 49:436-47.

13. Itandehui Ana, Pastrana EC, Rodríguez-Orozco R, Gómez C. Correlation between depression and family function in older adults from a Family Medicine Unit. Rev Méd Chile. 2009; 137:1642-3.

14. Díaz O, Soler M. APGAR Familiar en los Ancianos Convivientes. Revista Cubana Médica Integral. 1998; 14:548-53.

15. Peña B, Terán M, Moreno F, Bazán M. Auto percepción de la Calidad de Vida del Adulto Mayor en la Clínica de Medicina Familiar Oriente de ISSSTE. Revista de Especialidades Medico Quirúrgicas. 2009; 14:53-61.

16. Agudelo A, Martínez E. La salud bucal colectiva y el contexto colombiano: un análisis crítico. Revista Gerencia y Políticas de Salud. 2009; 8:91-105.

17. Pinzón S, Zunzunegui MV. Detección de necesidades de atención bucodental en ancianos mediante la autopercepción de la salud oral. Rev Mult Gerontol 1999; 9:216-24.

18. Maslow A. La personalidad Creadora: Teoría sobre la Motivación Humana. $9^{\mathrm{a}}$ ed. Barcelona: Kairós; 2008. p. 480.

19. Diez, J, Rivero ME, Alea A, García B. Impacto de la Salud Bucal en la Calidad de Vida. Instituto Superior de Ciencias Médicas de la Habana. Facultad de Estomatología. 2004; 3(8). Disponible en: http://www.ucmh.sld.cu/rhab/ reflexiones rev8.htm.

20. Arango V, Ruiz I. Diagnóstico de los Adultos Mayores de Colombia. Ministerio de Salud. 2001; 34 (1). Disponible en: http://www.saldarriagaconcha.org/Library/News/Files/ DIAGNOSTICO\%20ADULTO\%20MAYOR316.PDF.

21. Castrejon R. Salud Bucal en los Adultos Mayores y su Impacto en la Calidad de Vida. Instituto de Geriatría. 2007: 258-270. Disponible en: http://www.geriatria.salud.gob.mx/ descargas/26.pdf. 